\title{
Influencia de la asimetría de la marcha en el comportamiento biomecánico de las articulaciones de cadera en pacientes con prótesis transfemorales
}

\author{
Influence of gait asymmetry on hip joints biomechanical behavior in patients \\ with transfemoral prostheses
}

\author{
Leonardo Broche Vázquez ${ }^{1} \quad$ Mauricio Torres Quezada ${ }^{2} \quad$ Carlos Díaz Novo $^{3}$ \\ Pedro Pérez Bonne ${ }^{4} \quad$ Roberto Sagaró Zamora ${ }^{1}$ \\ Recibido 3 de abril de 2013, aceptado 30 de junio de 2014 \\ Received: April 3, 2013 Accepted: June 30, 2014
}

\begin{abstract}
RESUMEN
El presente trabajo establece la relación existente entre la asimetría en la marcha del amputado que emplea prótesis transfemoral pasiva y la aparición de las patologías de cadera en el miembro sano y el amputado. Este propósito se logra mediante una evaluación integradora de los resultados del análisis cinemático y dinámico de la marcha del amputado a través de técnicas videográficas y el empleo de ensayos clínicos como la radiografía y la densitometría, como el más importante aspecto relacionado con la evaluación del diseño y la funcionabilidad de la prótesis transfemoral.

Como resultado de la medición de las variables espacio-temporales practicadas a pacientes sanos y amputados se pudo establecer gran diferencia en los patrones de marcha entre la marcha de sujetos normales y pacientes amputados, así como entre los miembros sano y amputado de estos últimos. A través del análisis dinámico inverso (ADI) y con el empleo del método de los elementos finitos se pudo establecer que estos pacientes a partir de tal asimetría de la marcha experimentan un desequilibrio dinámico y que están relacionadas con enfermedades degenerativas de la cadera. Los ensayos clínicos practicados demostraron la prevalencia en estos pacientes de osteoporosis en el miembro amputado en 7 pacientes, el 87,5\% de los casos en estudio, lo que pone en evidencia la influencia en la remodelación ósea del estímulo mecánico y la presencia de osteoartritis en ambos miembros.
\end{abstract}

Palabras clave: Biomecánica, prótesis transfemoral, osteoporosis, osteoartritis, desequilibrio dinámico.

\section{ABSTRACT}

This study establishes the existing relation between gait asymmetry of an amputee that wears passive transfemoral prosthesis and the appearance of the hip pathologies in healthy and amputated limbs. This purpose is achieved through an integrated assessment of the results obtained out of kinematic and dynamic analysis of the amputee gait throughout the videographic techniques and the use of clinical trials such as radiography and densitometry, as the most important aspect related to design assessment and functionality of the transfemoral prosthesis.

The result of the spatiotemporal variables measurement performed on healthy and amputee patients established a significant difference between gait patterns of healthy and amputee patients, and between healthy and amputated limbs of the amputee. Dynamic inverse analysis (DIA) and implementation of finite element method demonstrated that patients with such gait asymmetry have dynamic imbalance related to the degenerative hip diseases. Performed clinical trials showed the prevalence of osteoporosis

1 Grupo Tribológico. Departamento de Mecánica y Diseño. Facultad de Ingeniería Mecánica. Universidad de Oriente. Santiago de Cuba, Cuba.E-mail: leo@fim.uo.edu.cu; sagaro2001@yahoo.com

2 Instituto Politécnico "Juan Bosco". Bogotá, D.C., Colombia. E-mail: torresqu_01@ hotmail.com

3 Centro Nacional de Biofísica Médica. Universidad de Oriente. Santiago de Cuba, Cuba. E-mail: cdiaznovo@yahoo.es

4 Laboratorio de Ortopedia Técnica. Santiago de Cuba, Cuba. E-mail: protesis@medired.scu.sld.cu 
in amputated limb of 7 patients, an $87.5 \%$ of studied cases, which highlights the influence of mechanical stimulus on bone remodeling and the presence of osteoarthritis in both limbs.

Keywords: Biomechanics, trans-femoral prosthesis, osteoporosis, osteoarthritis, dynamic imbalance.

\section{INTRODUCCIÓN}

El empleo de una o más prótesis en las personas con amputación transfemoral, representa una significativa alteración de la biomecánica de su aparato locomotor, lo cual viene justificado porque estas personas durante sus actividades diarias tienen como tendencia a recargar su miembro intacto, todo lo cual puede llevar en determinado lapso de tiempo a la aparición de patologías como es el caso de la osteoartritis de la rodilla y cadera del miembro sano. Consecuentemente con ello, la osteopenia y más tarde la osteoporosis ocurren como un posible efecto secundario debido al insuficiente estímulo mecánico relacionado por los procesos de remodelado óseo de los huesos largos de los miembros inferiores.

Estos aspectos pueden verse magnificados aún más por la selección inapropiada de la prótesis, aspecto este último que está relacionado con la edad, nivel de actividad, etc. La mala alineación de la prótesis, su peso y dimensiones, un deficiente trabajo de entrenamiento fisioterapéutico, son aspectos a considerar en estos pacientes. Sean cuales fueran las causas de estas asimetrías los esfuerzos que estos pacientes ejercen sobre el miembro sano exceden los valores de un sujeto normal, y por consiguiente la cadera amputada recibe un esfuerzo mecánico de menor cuantía [1-2].

Las estadísticas en la mayoría de aquellos países alejados de frecuentes guerras, exhiben índices que apuntan a niveles de amputación altos relacionados con accidentes o enfermedades vasculares, accidentes del tránsito y otros de índole genético en menor cuantía y en un buen porciento de estos pacientes tempranamente en su vida comienzan a emplear los artíficos protésicos.

Comoquiera que este trabajo da continuidad a trabajos precedentes de los autores [3-4] sigue la misma línea de pensamiento en cuanto a una evaluación integrada del diseño y la funcionabilidad de las prótesis transfemorales empleadas en Cuba. Así pues en su desarrollo se han tomado determinadas consideraciones tales como:

1. Adquisición de datos relacionados con la morfología del paciente, estructura muscular, del miembro residual. Utilización de herramientas de ingeniería inversa (ADI) bajo condiciones estáticas y dinámicas.

2. Modelado físico de cada una de las partes componentes, formas, dimensiones, materiales, etc.

3. Análisis cinemático y dinámico del ciclo de marcha empleando técnicas de videografía.

Estos además de establecer puntos de partida para los análisis de ADI permiten sacar conclusiones preliminares sobre patrones patológicos.

4. Aplicación del método de los elementos finitos (MEF)

5. Empleo de métodos clínicos como la radiografía y la densitometría.

\section{MÉTODOS}

\section{Caracterización de la marcha por técnicas videográficas}

Los estudios de caracterización de la marcha fueron practicados a pacientes en el Laboratorio de Análisis de Marcha del Centro de Investigaciones de Biofísica Médica en el hospital clínico quirúrgico de Santiago de Cuba siguiendo los protocolos establecidos al efecto [4-5].

Los pacientes objetos de estudio fueron 8 amputados transfemorales (AKA) que deambulaban con prótesis durante un tiempo nunca menor de 5 años (Tabla 1). Su edad osciló entre 43-83 años con altura y peso promedio $1,70 \mathrm{~m}$ y $80 \mathrm{~kg}$ de peso respectivamente. Antes de la realización de las mediciones todas las prótesis fueron revisadas minuciosamente. Todos ellos usaban encajes de tipo cuadrangular, rodillas protésicas del tipo POLIOR MO-01 y pie protésico modular articulado RA-10. Todas las mediciones fueron recogidas con arreglo al plano sagital. 
Como patrones o pacientes de control (CKA) se seleccionaron 6 pacientes respetando que estos tuvieran aproximadamente características anatómicas y edad muy similares. Cada paciente desarrolló para las mediciones una caminata libre de unos 4 metros aproximadamente con cadencia libre (Figura 1).

El ciclo de marcha fue registrado con tres cámaras CANON Zr 95 equipadas con un lente de $25 \mathrm{~mm}$ y luego transferido a un ordenador mediante el protocolo de comunicación IEEE 1394. Un promedio de 30 mediciones fueron realizadas para cada sujeto. El procesamiento de los resultados se realizó a través del paquete profesional HU-M-AN de HMA Technology Inc. [6].

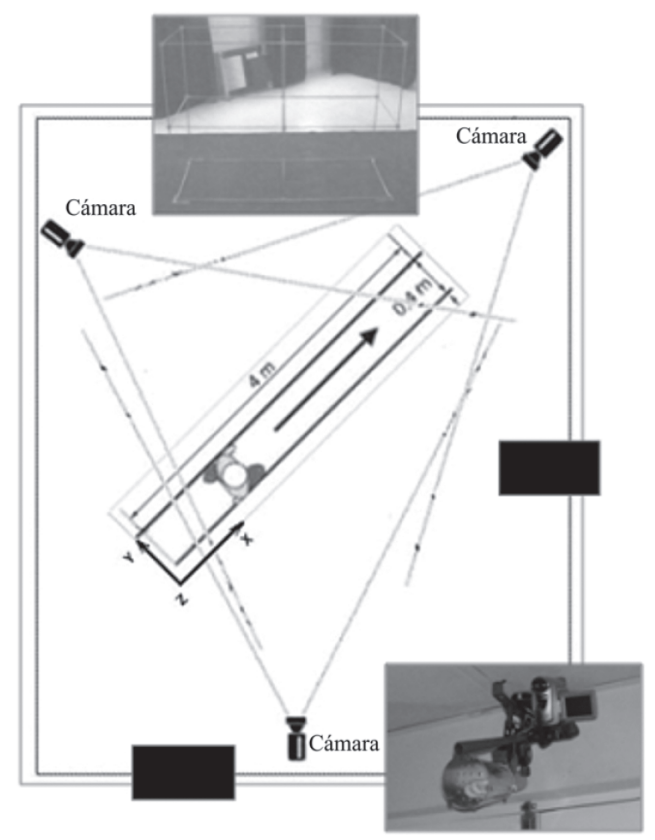

Figura 1. Técnica de Videografía.
Siguiendo el proceder experimental de otros trabajos precedentes $[4,7]$ se emplearon procederes establecidos por diversos investigadores (Zatsiorski y Seluyanov [8], Winter [9], Bresler y Frankel [10] y Braune [11]) para completar el análisis dinámico inverso (ADI). De igual forma, comoquiera que se trata de evaluar la influencia de la asimetría de la marcha en el comportamiento patológico de las caderas del amputado se consideró la determinación del ángulo de entrada del pie durante el apoyo del talón a partir de la determinación teórica de la longitud del paso y cuyo valor real puede determinarse con el empleo del paquete HU-MAN (Figura 2).

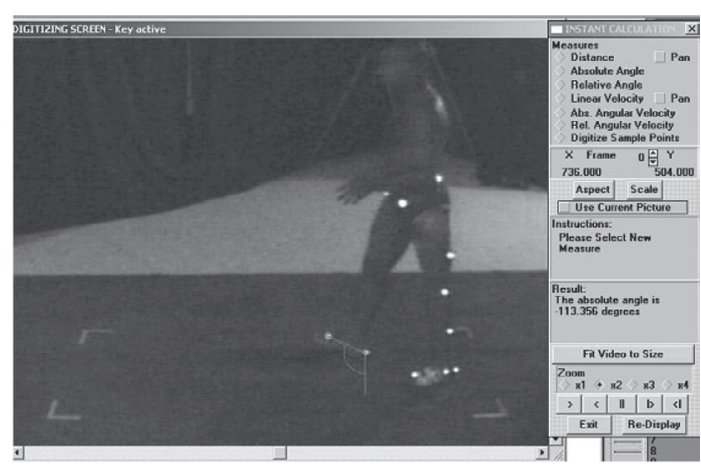

Figura 2. Determinación del ángulo de entrada de apoyo del talón según HU-MAN.

\section{Método de elementos finitos (MEF)}

El vínculo entre los datos tomados en los análisis de marcha y los análisis de tensiones aplicando el MEF se realiza calculando las fuerzas de reacción a que están sometidas las articulaciones de la prótesis transfemoral durante la marcha normal; para calcular estas magnitudes, se plantean las ecuaciones de dinámica vectorial, disponiendo de un conjunto de datos antropométricos (másicos e inerciales).

Tabla 1. Características de los pacientes.

\begin{tabular}{|c|c|c|c|c|c|c|c|c|}
\hline $\begin{array}{c}\text { Pacientes } \\
(*)\end{array}$ & $\begin{array}{c}\text { Edad } \\
(\mathbf{a n ̃ o s})\end{array}$ & $\begin{array}{c}\text { Años de } \\
\text { Amputación }\end{array}$ & $\begin{array}{c}\text { Uso } \\
\text { diario } \\
\text { (horas) }\end{array}$ & $\begin{array}{c}\text { Talla } \\
(\mathbf{m})\end{array}$ & $\begin{array}{c}\text { Peso } \\
(\mathbf{k g})\end{array}$ & $\begin{array}{c}\text { Peso de } \\
\text { pierna } \\
(\mathbf{k g})\end{array}$ & $\begin{array}{c}\text { Peso del } \\
\text { Muslo } \\
(\mathbf{k g})\end{array}$ & $\begin{array}{c}\text { Peso del } \\
\text { Pie } \\
(\mathbf{k g})\end{array}$ \\
\hline $1(\mathrm{I})$ & 83 & 24 & 4 & 1,68 & 78 & 3,26 & 11,06 & 0,99 \\
\hline $2(\mathrm{I})$ & 53 & 15 & 8 & 1,59 & 74 & 3,01 & 10,35 & 0,90 \\
\hline $3(\mathrm{I})$ & 46 & 27 & 10 & 1,67 & 78 & 3,25 & 11,05 & 0,99 \\
\hline $4(\mathrm{I})$ & 43 & 22 & 4 & 1,75 & 82 & 3,49 & 11,74 & 1,07 \\
\hline $5(\mathrm{I})$ & 79 & 20 & 4 & 1,66 & 78 & 3,24 & 11,03 & 0,98 \\
\hline $6(\mathrm{D})$ & 52 & 25 & 10 & 1,74 & 83 & 3,51 & 11,87 & 1,08 \\
\hline $7(\mathrm{D})$ & 47 & 16 & 6 & 1,73 & 81 & 3,43 & 11,57 & 1,05 \\
\hline $8(\mathrm{D})$ & 65 & 15 & 8 & 1,78 & 87 & 3,70 & 12,52 & 1,14 \\
\hline Promedio & 58 & 18 & 7 & 1,70 & 80 & 3,36 & 11,40 & 1,02 \\
\hline
\end{tabular}

*[Amputado de pierna I- izquierda, D-derecha]. 
El análisis dinámico inverso (ADI) se ha realizado para la posición de apoyo del talón como condición extrema. Se utiliza una simplificación mediante sólidos rígidos unidos mediante articulaciones simples y que permiten la rotación entre cada par de sólidos rígidos.

La modelación matemática por Elementos Finitos (MEF) ha sido ampliamente utilizada para modelar los elementos componentes de los artificios protésicos [12-14]. La modelación contempló el uso de las propiedades mecánicas del tejido óseo y los materiales blandos [7]. Todos los modelos fueron practicados con el empleo del paquete profesional SOLIDWORK 2011. Para los análisis se empleó el paquete profesional SOLIDWORK 2011, realizándose los análisis como sólidos bajos condiciones estables y lineales. Se utilizan elementos tetraédricos de segundo orden perfectamente adaptables a la complejidad de la geometría de la prótesis, para el acoplamiento encaje-zona biológica 65.350 tetraedros (Figura 3 ).

En la Tabla 2 se muestran las propiedades mecánicas de los materiales empleados en la modelación. En el caso del hueso fémur se consideró la conocida anisotropía del tejido óseo [15-16]. Se evalúa el estado tensional y los desplazamientos de las articulaciones de la prótesis teniendo en cuenta diferentes desviaciones angulares, así como los valores de las fuerza de reacción externas que fueron calculados mediante las ecuaciones de equilibrio dinámico.

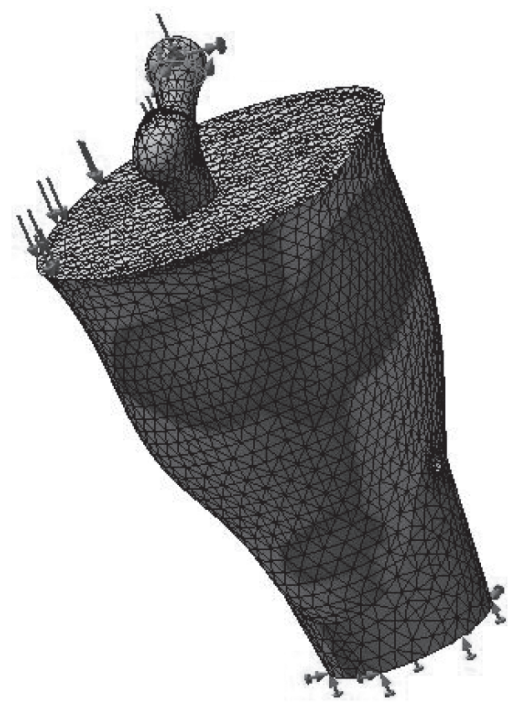

Figura 3. Mallado del modelo geométrico del sistema encaje-fémur-muñón.

\section{Densitometría}

La densitometría ósea es una técnica diagnóstica que permite medir la densidad mineral del hueso (BMD). El informe de los resultados de la densitometría ósea proporciona los valores promedios de masa ósea en cada zona explorada y medida, con imágenes digitalizadas y coloreadas para relacionar estos valores promedio con los de la normalidad en función de la edad y el sexo del paciente. Constituye la principal herramienta diagnóstica utilizada en la osteoporosis y de esta manera se puede determinar el riesgo de sufrir fracturas óseas. La realización

Tabla 2. Propiedades de los materiales empleados en la modelación.

\begin{tabular}{|c|c|c|c|c|c|}
\hline $\begin{array}{c}\text { Zona del } \\
\text { modelo }\end{array}$ & Material & $\begin{array}{l}\text { Módulo } \\
\text { elástico } \\
\text { (GPa)* }\end{array}$ & $\begin{array}{c}\text { Coeficiente de } \\
\text { Poisson }\end{array}$ & $\begin{array}{c}\text { Densidad } \\
\left(\mathrm{kg} / \mathrm{m}^{3}\right)\end{array}$ & $\begin{array}{l}\text { Límite } \\
\text { elástico } \\
\text { (MPa) }\end{array}$ \\
\hline Encaje & Polipropileno & 1,6 & 0,3 & 920 & 35 \\
\hline \multirow{3}{*}{ Fémur } & \multirow{3}{*}{ Hueso } & 22 & 0,37 & \multirow{3}{*}{1900} & \multirow{3}{*}{121} \\
\hline & & 12 & 0,222 & & \\
\hline & & 13,4 & 0,235 & & \\
\hline Piel, etc. & Tejidos blandos & $0,2 \cdot 10^{-3}$ & 0,4 & 1000 & 0,1 \\
\hline Pie Protésico & Poliuretano & 25 & 0,49 & 1200 & 30 \\
\hline Talón & Caucho & $6,1 \cdot 10^{3}$ & 0,49 & 1000 & $92,10^{6}$ \\
\hline $\begin{array}{l}\text { Tobillo } \\
\text { articular }\end{array}$ & Acero AISI 304 & $1,9 \cdot 10^{2}$ & 0,29 & 8000 & 206,8 \\
\hline
\end{tabular}


de las mediciones se practicó en el densitómetro marca Lexxos, de fabricación francesa y que presta servicios en las instalaciones de salud de la ciudad. Los resultados de la prueba generalmente se informan como "puntuación T".

- La puntuación T compara la densidad ósea del paciente con una persona de 30 años.

En cualquier puntuación, un número negativo significa que se tienen huesos más delgados que los estándares.

- Una puntuación T de -1 a -2,5 indica principio de pérdida ósea (osteopenia).

- Una puntuación T por debajo de -2,5 indica osteoporosis.

\section{Técnicas Radiográficas para la cadera}

Para la realización de las radiografías practicadas se siguieron los procedimientos de rutina para estos casos según la práctica clínica y fueron practicadas para la totalidad de los pacientes. Las técnicas empleadas fueron la de cadera frontal y la de cuellos femorales laterales comparativos (Figura 5) [17].

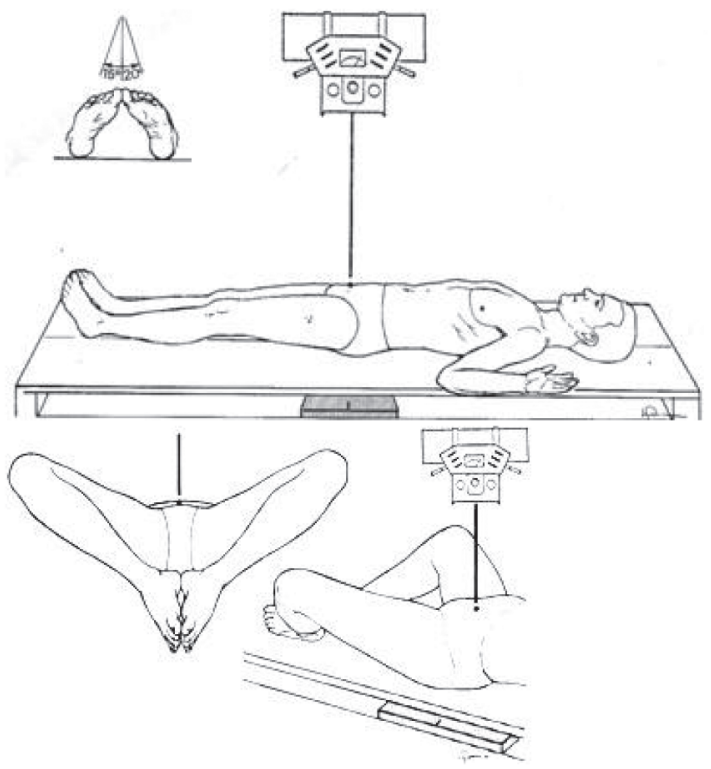

Figura 4. Posición para radiografiar cadera frontal y ambos cuellos femorales comparativos.

\section{RESULTADOS}

\section{Análisis de marcha}

La Tabla 3 refleja las mediciones de las variables espacio-temporales para los sujetos normales y amputados. La Figura 5 (a) muestra los resultados de los desplazamientos angulares promedio de los pacientes control y de los pacientes amputados promedios (Figura 5 (b)), considerando en este último caso tanto el miembro amputado como el intacto. En el caso del ángulo de entrada del pie al piso en los pacientes amputados analizados, de acuerdo a las medidas antropométricas de los pacientes, su valor durante el apoyo del talón debió ser de $13-15^{\circ}$, pero según las técnicas videográficas fue solo de $6^{\circ}$ como promedio.

Si comparamos la marcha fisiológica con la del amputado transfemoral saltan a la vista significativas diferencias.

Tales resultados corroboran aquellos anteriores de los autores y de otros investigadores y que apuntan a una significativa asimetría en la marcha del paciente amputado transfemoral con prótesis mecánicas $[4,7$, $18,19]$. De forma breve las principales alteraciones de la marcha pueden resumirse:

- Todas las articulaciones del miembro sano del paciente amputado describen similares trayectorias que las de pacientes sanos aunque con un mayor periodo de retardo y menor amplitud en algunos picos.

- La longitud del paso del miembro amputado es inferior a la del miembro intacto y la de los sujetos normales, lo que deriva en un ángulo de entrada al piso (Figura 2) muy pequeño (tobillo protésico con una exagerada flexión plantar respecto al sano) con una insuficiente extensión de la cadera.

- El paciente amputado se apoya durante la marcha más tiempo sobre su miembro sano. Esto conjuntamente con la anterior alteración conlleva a desequilibrio dinámico de la carga que actúa sobre ambas caderas del paciente amputado. Se observa una marcada asimetría en el patrón espacio-temporal de los respectivos desplazamientos angulares (cadera, la rodilla y tobillo) entre el miembro sano y el amputado.

- La cadera del amputado se mantiene en flexión durante toda la fase de apoyo. Se aprecia una ostensible disminución del rango de angular del desplazamiento de la cadera del 
Tabla 3. Variables espacio-temporales de la marcha.

\begin{tabular}{|c|c|c|c|c|c|c|}
\hline \multirow{2}{*}{ Variables } & \multicolumn{2}{|c|}{ Sujetos normales } & \multicolumn{3}{|c|}{ Pacientes amputados } \\
\cline { 3 - 7 } & \multicolumn{2}{|c|}{$\begin{array}{c}\text { Miembro } \\
\text { intacto }\end{array}$} & \multicolumn{2}{c|}{ Miembro protésico } \\
\cline { 2 - 7 } & Media & $\mathbf{D E}^{*}$ & Media & $\mathbf{D E}^{*}$ & Media & DE$^{*}$ \\
\hline $\begin{array}{c}\text { Velocidad de } \\
\text { la marcha (m/s) }\end{array}$ & 1,20 & 0,05 & 0,683 & 0,17 & 0,517 & 0,15 \\
\hline Longitud de Ciclo (m) & 1,24 & 0,07 & 1,031 & 0,15 & 0,976 & 0,11 \\
\hline Longitud de Paso (m) & 0,64 & 0,05 & 0,525 & 0,10 & 0,476 & 0,15 \\
\hline Tiempo de apoyo (s) & 0,89 & 0,07 & 1,156 & 0,19 & 0,836 & 0,08 \\
\hline Tiempo de Balanceo (s) & 0,53 & 0,15 & 0,459 & 0,18 & 0,576 & 0,19 \\
\hline
\end{tabular}

DE* Desviación estándar.
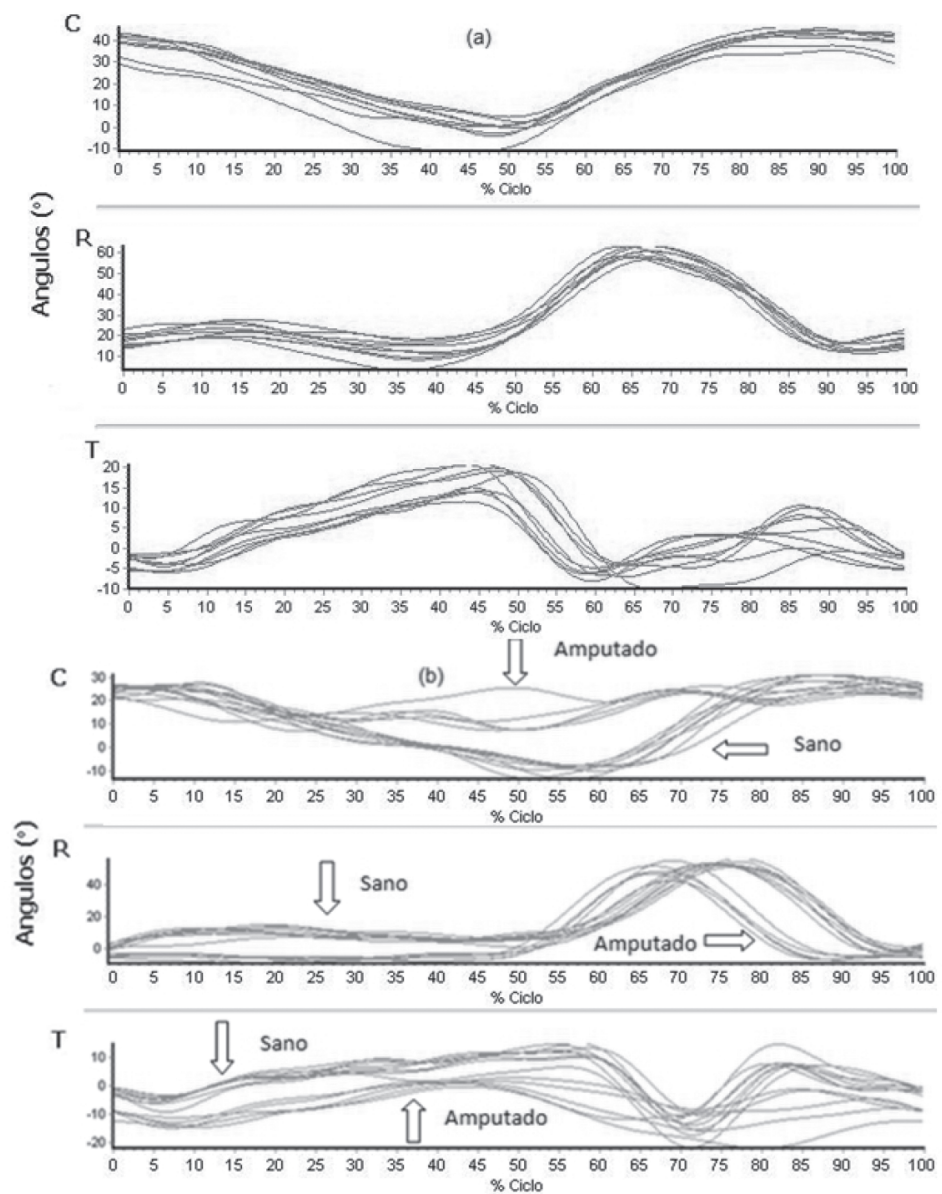

Figura 5. Resultados de los análisis de marcha. (a) Paciente promedio sano. (b) Amputado. 
miembro amputado respecto al de la sana, lo cual está relacionado con la existencia de un patrón temporal de compensación debido a la circunducción del miembro amputado durante la fase de balanceo para evitar así rozar el piso con la base del pie en la porción media de esta fase (balanceo). El escaso recorrido angular de la cadera está relacionado con esto y con una mayor oscilación lateral del tronco.

- La rodilla protésica permanece extendida durante toda la fase de apoyo para flexionarse durante el balanceo pero con 10 grados de antelación a la sana, lo que corrobora el menor tiempo de apoyo. Al no existir esta pequeña flexión de la rodilla, el choque dinámico se incrementa.

\section{Método de los elementos finitos}

La Figura 6 representa a manera de resumen una comparación de resultados en cuanto a estado tensional para la posición de contacto del talón con ángulo de entrada $6^{\circ}\left(\mathrm{M}=75\right.$ N.m) y $15^{\circ}(\mathrm{M}=107$ N.m). A manera de comentario se puede significar que el apoyo de talón con un ángulo de entrada de $15^{\circ}$ y con el peso actual del conjunto prótesis miembro amputado exigiría un consumo energético, considerando que no existen diferencias significativas en la velocidad del miembro sano y el residual, de 1,4 veces superior al desarrollado por los pacientes en análisis. Si bien como hemos comentado con anterioridad no existe unanimidad respecto al aspecto relacionado con el consumo metabólico y el peso de los artificios protésicos, existe sin embargo consenso general que las alteraciones en la marcha aquí analizadas y la consecuente sobrecarga adicional en el miembro sano del amputado significa el peligro de la aparición de patologías como es el caso de la osteoartritis y en el miembro residual osteopenia y la subsecuente osteoporosis como resultado de insuficientes estímulos mecánicos, todo lo cual ha sido reportado por una gran número de investigaciones [7, 18, 20-21].

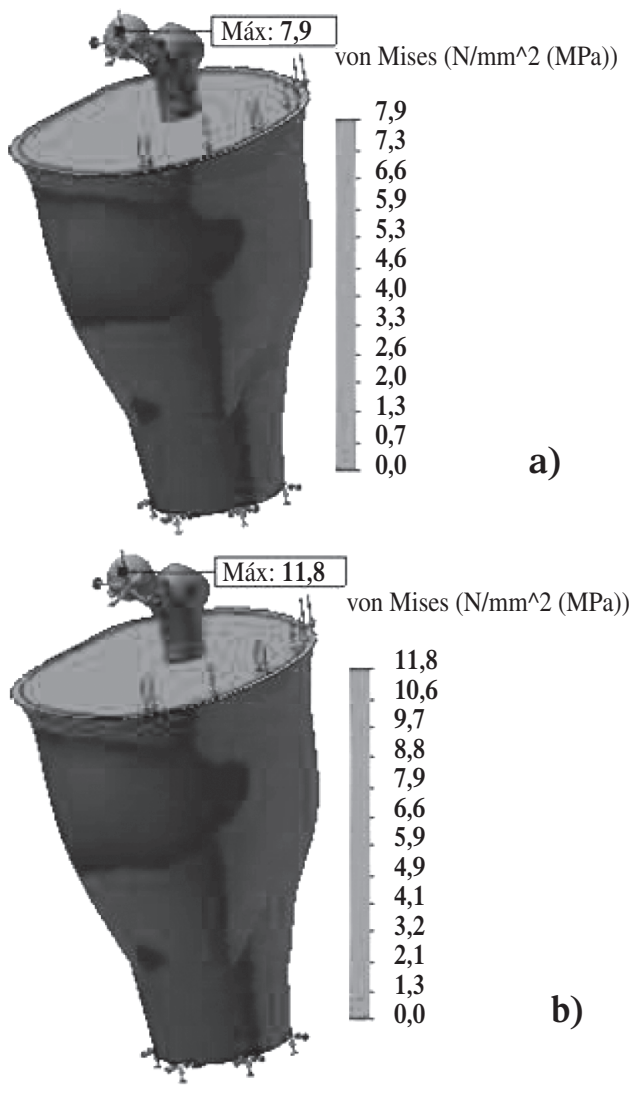

Figura 6. Distribución de tensiones en el conjunto encaje-muñón-fémur, (a) ángulo de entrada $6^{\circ}$, (b) $15^{\circ}$. 


\section{Ensayos de densitometría}

Los resultados practicados a los pacientes se muestran en las Tablas 4 y 5 .

Como se puede observar en la Tabla 4 existen significativas diferencias en los pacientes amputados en la densidad ósea del miembro amputado y el intacto, que son más notables en unas zonas que en otras. La Tabla 5 indica los valores del índice $\mathbf{T}$ score.

La prevalencia de los fenómenos de osteoartritis, osteopenia y osteoporosis en ambos grupos de sujetos (sanos y amputados) se recoge en la Tabla 6.

De la Tabla 6 se infiere que un número importante de pacientes amputados $5(62,5 \%)$ presentaron osteoporosis en algún cuerpo vertebral (L1L4), mientras 2 (25\%) pacientes presentaron osteopenia. En tanto en los pacientes control 1 (16\%) presentó osteopenia y $3(50 \%)$ osteoporosis. En la cadera sin embargo la BMD de los pacientes amputados fue significativamente menor para el miembro amputado que para el sano, todo lo cual consideramos sea responsabilidad en gran medida de la prolongada disminución del efecto mecánico de la distribución de las cargas como resultado de una marcha asimétrica, cuyas características han sido ampliamente debatidas por sus autores [1-2, 20-22]. De los resultados se concluyó que 7 de los pacientes amputados presentaron osteoporosis en la cadera $(\mathbf{8 7 , 5 \%})$ en el miembro amputado, siendo la zona inter trocánter la más notable.

La Figura 7 es representativa de lo comentado para un paciente amputado al que se le han practicado ensayos de densitometría en ambos miembros.

Tabla 4. Valores de la densidad ósea para los pacientes (AKA).

\begin{tabular}{|c|c|c|c|c|c|c|c|c|c|}
\hline \multirow[t]{2}{*}{$\begin{array}{l}\text { Paciente } \\
(*)\end{array}$} & \multirow[t]{2}{*}{$\begin{array}{c}\text { Total } \\
\text { Columna } \\
\text { BMD } \\
\left(\mathrm{g} / \mathrm{cm}^{2}\right)\end{array}$} & \multicolumn{2}{|c|}{$\begin{array}{l}\text { Cadera Total } \\
\operatorname{BMD}\left(\mathrm{g} / \mathrm{cm}^{2}\right)\end{array}$} & \multicolumn{2}{|c|}{$\begin{array}{c}\text { Cuello } \\
\text { femoral } \\
\mathrm{BMD}\left(\mathrm{g} / \mathrm{cm}^{2}\right)\end{array}$} & \multicolumn{2}{|c|}{$\begin{array}{l}\text { G. Trocánter } \\
\text { del femoral } \\
\operatorname{BMD}\left(\mathbf{g} / \mathbf{c m}^{2}\right)\end{array}$} & \multicolumn{2}{|c|}{$\begin{array}{c}\text { Inter } \\
\text { Trocánter } \\
\text { del femoral } \\
\text { BMD }\left(\mathrm{g} / \mathrm{cm}^{2}\right)\end{array}$} \\
\hline & & A & NA & A & NA & A & NA & A & NA \\
\hline 1(I) & 0,795 & 0,29 & 0,69 & 0,36 & 0,60 & 0,27 & 0,55 & 0,28 & 0,75 \\
\hline 2(I) & 1,06 & 0,80 & 1,08 & 0,68 & 1,16 & 0,51 & 0,83 & 0,88 & 1,14 \\
\hline 3(I) & 1,052 & 0,82 & 1,23 & 0,88 & 1,07 & 0,66 & 0,88 & 0,88 & 1,32 \\
\hline 4(I) & 1,05 & 0,79 & 1,06 & 0,62 & 1,18 & 0,51 & 0,83 & 1,06 & 1,12 \\
\hline 5(I) & 0,80 & 0,40 & 0,70 & 0,42 & 0,65 & 0,35 & 0,60 & 0,32 & 0,78 \\
\hline 6(D) & 1,061 & 0,80 & 1,20 & 0,86 & 1,05 & 0,62 & 0,85 & 0,84 & 1,30 \\
\hline 7(D) & 1,06 & 0,75 & 1,02 & 0,60 & 1,08 & 0,50 & 0,72 & 0,75 & 1,10 \\
\hline 8(D) & 1,062 & 0,60 & 1,05 & 0,70 & 1,08 & 0,65 & 0,85 & 0,72 & 1,08 \\
\hline
\end{tabular}

*[Amputado de pierna I- izquierda, D-derecha]. A-lado amputado, NA-miembro sano.

Tabla 5. Índice T score para los pacientes amputados analizados (AKA).

\begin{tabular}{|c|c|c|c|c|c|c|c|c|c|}
\hline \multirow[t]{2}{*}{$\begin{array}{l}\text { Pacientes } \\
(*)\end{array}$} & \multirow[t]{2}{*}{$\begin{array}{c}\text { Total } \\
\text { Columna } \\
\text { T score }\end{array}$} & \multicolumn{2}{|c|}{$\begin{array}{l}\text { Cadera Total } \\
\text { T score }\end{array}$} & \multicolumn{2}{|c|}{$\begin{array}{c}\text { Cuello } \\
\text { femoral } \\
\text { T score }\end{array}$} & \multicolumn{2}{|c|}{$\begin{array}{c}\text { G. } \\
\text { Trocánter } \\
\text { del femoral } \\
\text { T score }\end{array}$} & \multicolumn{2}{|c|}{$\begin{array}{c}\text { Inter } \\
\text { Trocánter } \\
\text { del femoral T } \\
\text { score }\end{array}$} \\
\hline & & A & NA & $\mathbf{A}$ & NA & $\mathbf{A}$ & NA & $\mathbf{A}$ & NA \\
\hline 1(I) & $-3,4$ & $-5,3$ & $-3,1$ & $-4,0$ & $-2,5$ & $-4,6$ & $-2,6$ & $-6,0$ & $-3,3$ \\
\hline 2(I) & $-1,0$ & $-2,5$ & $+0,9$ & -2 & $+1,6$ & $-2,8$ & $-0,7$ & $-2,5$ & $-1,1$ \\
\hline 3(I) & $-1,1$ & $-2,2$ & $-0,0$ & $-0,8$ & $-0,3$ & $-1,8$ & $-0,3$ & $-2,5$ & $+0,0$ \\
\hline 4(I) & $-1,1$ & $-2,6$ & $-0,7$ & $-2,5$ & $+0,4$ & $-2,5$ & $-0,7$ & $-2,9$ & $-1,2$ \\
\hline 5 (I) & $-3,3$ & $-4,6$ & $-3,1$ & $-3,8$ & $-2,3$ & $-4,0$ & $-2,5$ & $-5,3$ & $-3,0$ \\
\hline 6(D) & -1 & $-2,5$ & $-0,7$ & $-0,9$ & $+0,9$ & $-1,9$ & $-0,2$ & $-2,8$ & $+0,0$ \\
\hline 7(D) & $-1,1$ & $-2,8$ & $-0,8$ & $-2,8$ & $+0,0$ & $-2,5$ & $-0,9$ & -3 & $-1,4$ \\
\hline $8(\mathrm{D})$ & $-0,9$ & -3 & $-0,7$ & $-1,8$ & $+0,9$ & $-1,8$ & $-0,2$ & $-3,2$ & $-1,6$ \\
\hline
\end{tabular}

Osteopenia Osteoporosis A-miembro amputado NA-miembro no amputado. 
Tabla 6. Prevalencia de osteoartritis, osteoporosis y osteopenia en AKA (amputados) y CKA (control).

\begin{tabular}{|c|c|c|}
\hline Región & $\begin{array}{c}\text { AKA } \\
\text { Amputado No } \\
\text { amputado }\end{array}$ & $\begin{array}{c}\text { CKA } \\
\text { Amputado No } \\
\text { amputado }\end{array}$ \\
\hline $\begin{array}{c}\text { Total cadera } \\
\text { Osteopenia } \\
\text { Osteoporosis }\end{array}$ & 10 & 01 \\
Osteoartritis & 72 & 00 \\
\hline Columna & 54 & 00 \\
Osteopenia & 2 & 1 \\
Osteoporosis & 5 & 3 \\
\hline Cuello femoral & 22 & 11 \\
Osteopenia & 41 & 00 \\
Osteoporosis & & \\
\hline G.Trocánter del & & 22 \\
femoral & 30 & 00 \\
Osteopenia & 52 & 22 \\
Osteoporosis & & 00 \\
\hline Inter Trocánter & & \\
del femoral & & \\
Osteopenia & & \\
Osteoporosis & & \\
\hline
\end{tabular}

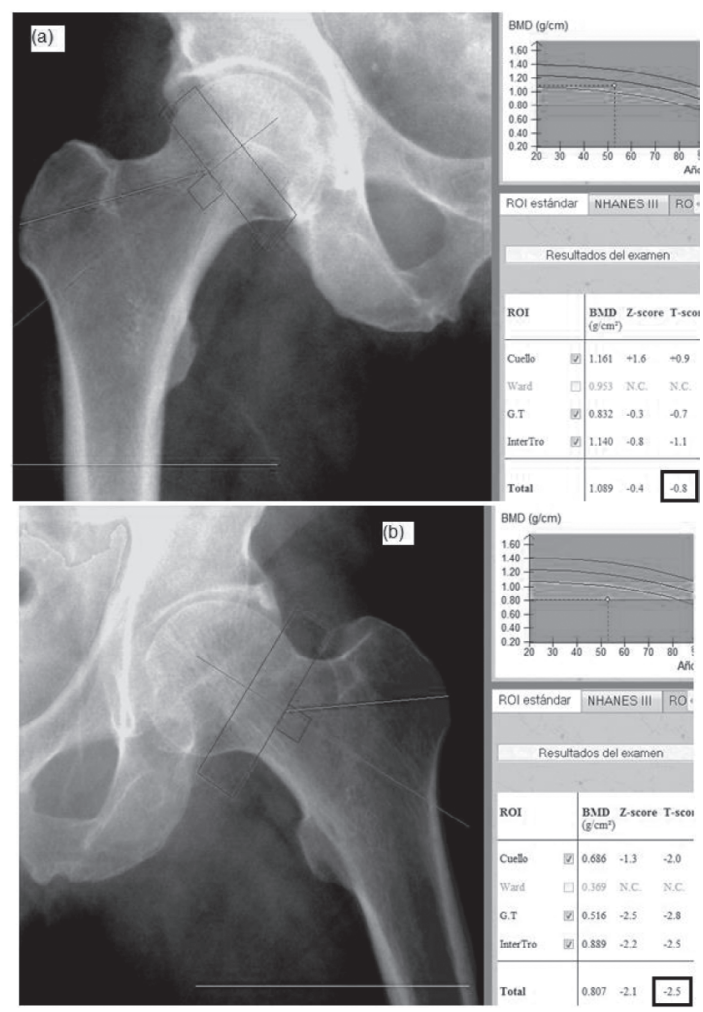

Figura 7. Resultado del ensayo de densitometría para un paciente de 53 años con 15 de amputación. (a) Miembro intacto (normal T=-0,8). (b) Miembro amputado (osteoporosis moderada, $\mathrm{T}=-2,5$ ).

\section{Técnicas de radiografía de cadera}

Se emplearon fundamentalmente para buscar indicios de la existencia de osteoartritis en ambos miembros que pudieran estar asociados a la amputación y deambulación del paciente amputado. La Figura 8 muestra los resultados para un paciente de 83 años, 20 años de amputación. Como se puede observar este paciente presenta una pérdida del espacio articular en ambas caderas sintomático de una osteoartritis avanzada y que ya manifiesta en el paciente dolores y rigidez en las articulaciones.

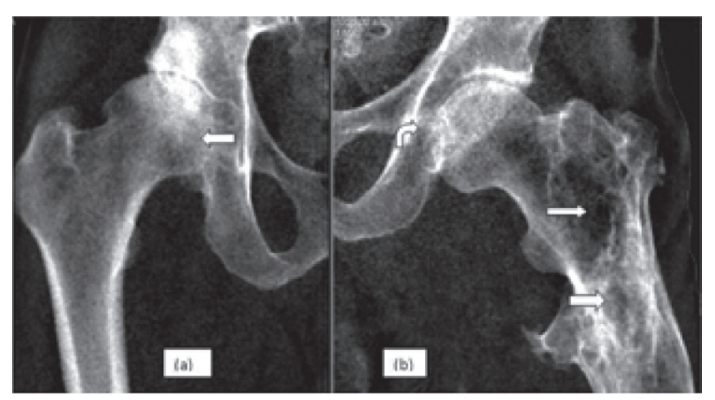

Figura 8. Radiografías de ambas caderas de un paciente amputado de 82 años con claros signos de osteoartritis avanzada en ambas caderas. (a) Cadera sana. (b) cadera amputada.

Aunque no resulte concluyente la comparación, pues otros factores además de la edad y los efectos de la asimetría cinemática y dinámica de la marcha algunos de los pacientes control con una edad muy aproximada no presentaron daño alguno en ambas caderas. Este amputado aunque contrasta por estos elementos con el resto y que quizás no lo conviertan en el caso más ilustrativo, sin embargo muestra un tendencia que quisiéramos significar y que se muestra en la Figura 9 para un paciente amputado de 43 años con 20 años de amputación y actividad diaria intensa.

Como se puede observar este paciente presenta síntomas de osteoartritis muy prematura en la cadera intacta y sin embargo un daño de consideración en la cadera del miembro residual. Como se puede observar en el detalle existe una destrucción parcial de la zona articular en esta última con deformidad remante y osteofitosis, que en la muestra analizada puede considerarse un denominador común.

Este resultado puede parecer un tanto contradictorio por cuando dado el incremento de la carga que 


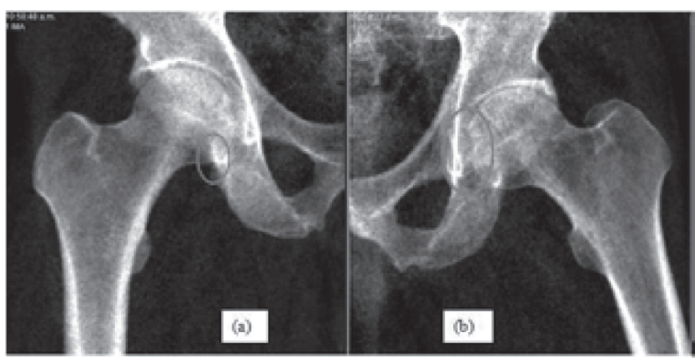

Figura 9. Imágenes radiográficas de ambas caderas de un paciente amputado de 43 años, 20 años de amputación. (a) Cadera intacta. (b) Cadera miembro amputado.

asimila la cadera intacta era de esperar un posible aumento de las presiones articulares y destrucción del cartílago articular. No obstante tal comportamiento ha sido repostado por destacados investigadores [2, 21, 23-25]. Tal situación parece estar relacionada con los desajustes cinemáticos y dinámicos que experimenta el paciente durante la marcha.

\section{CONCLUSIONES}

Los análisis de la marcha del amputado permiten establecer diferencias significativas en el patrón de la marcha respecto a patrones sanos y entre el miembro sano y el amputado en pacientes con amputación transfemoral. Las principales diferencias en el miembro amputado están el ángulo de entrada al suelo $\left(\sim 6^{\circ}\right)$, la rodilla totalmente extendida durante la fase de apoyo y la cadera en flexión durante todo el ciclo de marcha. Es de significar que el paciente amputado durante la marcha se apoya más tiempo sobre su miembro sano que sobre el amputado y es de suponer, que la fuerza ejercida sobre la extremidad intacta sea mayor que sobre el residual y la que ejercería una persona sana sobre sus miembros inferiores durante la marcha normal.

A partir de los resultados del análisis de marcha, los resultados del ADI y la simulación por elementos finitos apuntan a un desbalance dinámico que ha sido reportado por diversos investigadores y que señalan al miembro remanente recibiendo durante la marcha un estímulo mecánico 1,5 veces menor que el miembro sano. La sobrecarga en el miembro sano del amputado significa la aparición de enfermedades como la osteoartritis; también en el miembro residual se puede encontrar la osteopenia y debido a las transferencias de cargas al miembro sano, aparecerán zonas osteoporóticas en consecuencia a la ausencia de estímulos mecánicos.

Los ensayos de densitometría practicados a los pacientes arrojaron notables diferencias con los pacientes control y entre los miembros amputado e intacto que definitivamente están asociados con la asimetría de la marcha, el tiempo de amputación de los pacientes y el tiempo de uso del artificio protésico. En el 87,5\% de los casos analizados se pudo verificar la existencia de osteoporosis, siendo esta más generalizada en la muestra de la cadera en la zona entre los trocánter mayor y menor del fémur del miembro remanente.

Los ensayos de radiología mostraron de igual forma la presencia de osteoartritis en los pacientes amputados, aunque sorpresivamente en estado avanzado en el miembro amputado de los pacientes y con signos de muy poco desarrollo en el miembro intacto de los pacientes. El fenómeno parece estar asociado a la ausencia de extensión en la cadera como resultado del funcionamiento asimétrico del artificio protésico y que al presentarse repetitivamente en el tiempo puede inducir lesiones articulares.

\section{AGRADECIMIENTOS}

Al Laboratorio de Ortopedia Técnica de Santiago de Cuba y a los pacientes amputados, sin su participación no hubiera sido posible. A los Hospitales clínicoquirúrgico Juan Bruno Zayas y al Hospital Militar de Santiago de Cuba por su inestimable colaboración.

\section{REFERENCIAS}

[1] R. Gailey. "Review of secondary physical conditions associated with lower-limb amputation and long-term prosthesis use". Journal of Rehabilitation Research \& Development. Vol. 45, Issue 1, pp. 15-30. 2008.

[2] J. Kulkarni, J. Adams, E. Thomas and A. Silman. "Association between amputation, arthritis and osteopenia in British male war veterans with major lower limb amputations". Clin Rehabil. Vol. 12, Issue 4, pp. 348-53. 1998.

[3] R. Sagaró. "Diseño de prótesis transfemorales". Memorias de la Red Académica Productos industriales: Diseño, fabricación y 
comunicación. Universidad de Vigo. España. ISBN: 84-8158-231-X. 2001.

[4] A. Olivares y R. Sagaró. "Evaluación integral de prótesis ortopédicas transfemorales". Revista Ingenierías. Vol. XIII $\mathrm{N}^{\circ} 47$, pp. 17-23. Universidad Autónoma de Nuevo León. México. Abril-Junio, 2010.

[5] C. Novo. "Caracterización cinemática de la motricidad en la ataxia espinocerebeloza hereditaria". Memorias del III Congreso latinoamericano de Ingeniería Biomédica. La Habana, Cuba. ISBN: 950-7132-57-5. 2001.

[6] Hu-m-an. Diciembre 2009. URL: http:// www.hma-tech.com/page01a.htm

[7] L. Broche y R. Sagaró. "Análisis de la funcionabilidad de prótesis ortopédicas transfemorales". Revista cubana de Ortopedia y Traumatología. Vol. 25 N² 2, pp. 102-116. 2011.

[8] V. Zatsiorski. "Biomecánica del Ejercicio Físico". Editorial Pueblo y Educación, pp. 76-92. La Habana, Cuba. ISBN: 5- 05001279-I. 1988.

[9] D. Winter. "Biomechanics and Motor Control of Human Movement". $2^{\text {nd }}$ edition. John Wiley \& Sons. New York, USA. 1990.

[10] B. Bresler. "The forces and moments in the leg during walking". ASME 72, pp. 27-36. 1950.

[11] W. Braune. "Determination of body moments of inertia of the human body and its limbs". Springer Verlag. 1988.

[12] S. Radi. "Analysis of a below knee prosthetic socket". Journal of Engineering and development. Vol. 12, Issue 2. 2008. ISSN: 1813-7822.

[13] M. Faustini. "The quasi- static response of compliant prosthetic sockets for transtibial amputees using finite element methods". Medical Engineering \& Physics. Vol. 28, pp. 114-121. 2006.

[14] F. Frillici and C. Rizzi. "A computer assisted methodology to innovate the development process of prosthesis socket". Research in interactive design. Springer ltd, pp. 23-41. Paris, Francia. 2007.
[15] M. Comin, J.L. Peris, J.M. Prat, J.R. Dejoz y P.M. Vera. "Biomecánica de la fractura ósea y técnicas de reparación". Instituto de Biomecánica de Valencia, pp. 33-72. Valencia, España. ISBN: 84-923974-5-4. 1999.

[16] R.B. Ashman. "Elastic moduli of trabecular bone material". Journal of Biomechanic. Vol. 21, pp. 177-181. 1988.

[17] J. Mulkay Moreno. "Rayos X". Editorial Pueblo y Educación, pp. 65-78. La Habana, Cuba. SNLC. CU 01.28403.7. 1987.

[18] F. Farahmand. "Kinematic and Dynamic Analysis of the gait cycle of above knee amputees". Scientia Iranica. Vol. 13, Issue 3, pp. 261-271. July, 2006.

[19] H. Van der Linden, C.J. Hofstad, A.C.H. Geurts and K. Postema. "A systematic literatura review of the effect of different prosthetic components on human functioning with a lower limb prosthesis". Journal of Rehabilitation Research \& Development. Vol. 41, Issue 4, pp. 555-70. 2004.

[20] J. Kulkarni. "Post amputation syndrome. Prosthetics and Orthotics International". Vol. 32, Issue 4, pp. 434-437. 2008.

[21] E.D. Lemaire and R.F. Fisher. "Osteoarthritis and elderly amputee gait". Arch. Phys. Med. Rehabil. Vol. 75, Issue 10, pp. 1094-1099. 1994.

[22] V.D. Sherk. "BMD and Bone Geometry in Transtibial and Transfemoral Amputees". Journal of bone and mineral research. Vol. 23, Issue 9, pp. 1149-1157. 2008.

[23] D.S. Hungerford and J. Cockin. "Fate of the retained lower limb joints in Second World War amputees". Proceedings and Reports of Universities, Colleges, Councils, and Associations. J Bone Joint Surg. Vol. 57 (B1), pp. 111-117. 1975.

[24] P.J. Rush, J.S. Wong, J. Kirsh and M. Devlin. "Osteopenia in patients with above knee amputation”. Arch. Phys. Med. Rehabil. Vol.75, Issue 1, pp. 112-115. 1994.

[25] M.J. Burke. "Bone and joint changes in lower limb amputees". Annals of the Rheumatic Diseases. Vol. 37, pp. 252-254. 1978. 